\title{
The Method of Coupled Fixed Points and Coupled Quasisolutions When Working with ODE's with Arguments of Bounded Variation
}

\author{
Rubén Figueroa ${ }^{1,2}$ \\ ${ }^{1}$ Department of Mathematical Analysis, University of Santiago de Compostela, 15782 Santiago de Compostela, Spain \\ ${ }^{2}$ Department of Specific Didactics, University of Burgos, 09001 Burgos, Spain
}

Correspondence should be addressed to Rubén Figueroa; ruben.figueroa.sestelo@gmail.com

Received 14 May 2013; Revised 2 October 2013; Accepted 4 October 2013

Academic Editor: Marcia Federson

Copyright (C) 2013 Rubén Figueroa. This is an open access article distributed under the Creative Commons Attribution License, which permits unrestricted use, distribution, and reproduction in any medium, provided the original work is properly cited.

\begin{abstract}
The aim of this paper is to show the use of the coupled quasisolutions method as a useful technique when dealing with ordinary differential equations with functional arguments of bounded variation. We will do this by looking for solutions for a first-order ordinary differential equation with an advanced argument of bounded variation. The main trick is to use the Jordan decomposition of this argument in a nondecreasing part and a nonincreasing one. As a necessary step, we will also talk about coupled fixed points of multivalued operators.
\end{abstract}

\section{Introduction}

In the paper [1], we proved a new result on the existence of coupled fixed points for multivalued operators, and then we used it to guarantee the existence of coupled quasisolutions and solutions to a certain first-order ordinary differential equation with state-dependent delay. In that paper, the nonlinearity was allowed to have both nondecreasing and nonincreasing arguments and the existence of solutions was obtained under strong Lipschitz conditions. We pointed out there that this tool could be useful when working with arguments of bounded variation, but no literature about this was written since then. So, the main goal in the present paper is to develop the application of the coupled quasisolutions technique in the framework of arguments of bounded variation, and we do it in an appropriate way, in order to take advantage of the Jordan decomposition and avoid the use of strong assumptions, as Lipschitz-continuity.

To show the application of this technique, we will study throughout this paper the existence of solutions for the following first-order problem:

$$
\begin{gathered}
x^{\prime}(t)=f(t, x(t), x(\tau(t))), \quad \text { for a.a. } t \in I=[a, b], \\
x(t)=\phi(t), \quad \forall t \in[b, b+r],
\end{gathered}
$$

where $r \geq 0, \tau$ is a measurable function such that $\tau(t) \geq t$ for a.a. $t$; that is, $\tau$ is an advanced argument, and $\phi$ is a bounded function which represents the final state of the solution. By a solution of (1), we mean a function $x \in \mathscr{C}[a, b+r]$ such that $x_{\mid I} \in A C(I)$ and $x$ satisfies both the differential equation (a.e. on $I$ ) and the final condition. We refer the readers to papers [2-4] to see more results on the existence of solutions and some applications of first-order problems with advance.

This paper is organized as follows. In Section 2, we gather some preliminary concepts and results involving functions of bounded variation and coupled fixed points of multivalued operators. These preliminaries are used later, in Section 3, to prove the existence of quasisolutions and solutions for problem (1). In Section 4, we show how our results can be adapted to deal with delay problems. Finally, in Section 5, some examples of application are available.

\section{Preliminaries on Bounded Variation and Coupled Fixed Points of Multivalued Operators}

In this section, we introduce some preliminaries that we will use throughout this work. First, we remember some concepts 
about functions of bounded variation. The reader can see more about this in the monographs $[5,6]$.

Definition 1. Given a function $f: I=[a, b] \subset \mathbb{R} \rightarrow \mathbb{R}$ and a partition $P=\left\{x_{0}, \ldots, x_{n}\right\}$ of $I$, one defines the variation of $f$ relative to the partition $P$ as the number

$$
V(f, P)=\sum_{i=1}^{n}\left|f\left(x_{i}\right)-f\left(x_{i-1}\right)\right|,
$$

and one defines the total variation of $f$ on $I$ as

$$
V_{a}^{b}(f)=\sup _{P \in \mathscr{P}} V(f, P)
$$

where $\mathscr{P}=\{P: P$ is a partition of $I\}$.

One says that $f$ is a function of bounded variation on $I$ if $V_{a}^{b}(f)<+\infty$. In that case, one writes $f \in \mathrm{BV}(I)$.

Functions of bounded variation satisfy the following wellknown result, which becomes essential now for our purposes.

Proposition 2 (Jordan decomposition). A function $f$ is of bounded variation on I if and only if there exist a nondecreasing function, $g$, and a nonincreasing one, $h$, such that

$$
f(t)=g(t)+h(t), \quad \forall t \in I .
$$

The proof of Proposition 2 uses the fact that the function $t \in I \rightarrow V_{a}^{t}(f)$ is nondecreasing and $t \rightarrow f(t)-V_{a}^{t}(f)$ is nonincreasing, and thus the desired decomposition is

$$
f(t)=V_{a}^{t}(f)+f(t)-V_{a}^{t}(f) .
$$

We remark that this decomposition is not unique. Finally, notice that, as a consequence of this result, every function of bounded variation is a.e. differentiable.

The set $\mathrm{BV}(I)$ is an algebra which is included neither in the set of continuous functions nor in its complementary. Indeed, if $f$ is monotone on $[a, b]$, then $V_{a}^{b}(f)=|f(b)-f(a)|$, and thus $f \in \operatorname{BV}(I)$. Then, there exist discontinuous functions which are of bounded variation (e.g., step function). In fact, it is also a well-known fact that if $f \in \mathrm{BV}(I)$, then $f$ has only "jump" discontinuities. On the other hand, there exist continuous functions which are not of bounded variation, as (see [5, Example 6.3.1])

$$
f(t)= \begin{cases}t \cos \left(\frac{\pi}{2 t}\right) & \text { if } 0<t \leq 1 \\ 0 & \text { if } t=0\end{cases}
$$

To obtain our main result, we will use a generalized monotone method in presence of lower and upper solutions. This is a very well-known tool which is extensively used in the literature of ordinary differential equations. The classical version of this technique uses a pair of monotone sequences which will converge to the extremal solutions of the problem. The generalized version of this technique was developed in [7], and it is used when the nonlinearity has discontinuous arguments and therefore the pair of monotone sequences is replaced by a monotone operator. As a novelty which respect to the method developed in [7] and related references, we will use here a multivalued operator (i.e., a set-valued mapping) defined in a product space and then we will look for coupled fixed points. We concrete this idea in the following lines.

Definition 3. A metric space $X$ equipped with a partial ordering $\leq$ is an ordered metric space if the intervals $[x)=$ $\{y \in X: x \leq y\}$ and $(x]=\{y \in X: y \leq x\}$ are closed for every $x \in X$. Let $P$ be a subset of an ordered metric space. An operator $A: P \times P \rightarrow P$ is said to be mixed monotone if $A(\cdot, x)$ is nondecreasing and $A(x, \cdot)$ is nonincreasing for each $x \in P$. One says that $A$ satisfies the mixed monotone convergence property (m.m.c.p.) if $\left(A\left(v_{j}, w_{j}\right)\right)_{j=1}^{\infty}$ converges in $X$ whenever $\left(v_{j}\right)_{j=1}^{\infty}$ and $\left(w_{j}\right)_{j=1}^{\infty}$ are sequences in $P$, one being nondecreasing and the other nonincreasing.

Definition 4. Let $\bar{X}$ be a subset of an ordered metric space $X$. One defines a multivalued operator in the product $\bar{X} \times \bar{X}$ as a mapping

$$
\mathscr{A}: \bar{X} \times \bar{X} \longrightarrow 2^{\bar{X}} \backslash \emptyset .
$$

We say that $v, w \in \bar{X}$ are coupled fixed points of $\mathscr{A}$ if $v \in$ $\mathscr{A}(v, w)$ and $w \in \mathscr{A}(w, v)$. We say that $v_{*}, w^{*} \in \bar{X}$ are the extremal coupled fixed points of $\mathscr{A}$ in $\bar{X}$ if $v_{*}, w^{*}$ are coupled fixed points of $\mathscr{A}$ and if $v, w \in \bar{X}$ are another pair of coupled fixed points of $\mathscr{A}$; then $v_{*} \leq v$ and $w \leq w^{*}$.

Theorem 5 (see [1, Theorem 2.1]). Let $Y$ be a subset of an ordered metric space $X,[\alpha, \beta]$ be a nonempty closed interval in $Y$, and $\mathscr{A}:[\alpha, \beta] \times[\alpha, \beta] \rightarrow 2^{[\alpha, \beta]} \backslash \emptyset$ be a multivalued operator.

If for all $v, w \in[\alpha, \beta]$, there exist

$$
\begin{aligned}
& A_{*}(v, w)=\min \mathscr{A}(v, w) \in[\alpha, \beta], \\
& A^{*}(v, w)=\max \mathscr{A}(v, w) \in[\alpha, \beta],
\end{aligned}
$$

and the (single-valued) operators $A_{*}$ and $A^{*}$ are mixed monotone and satisfy the m.m.c.p., then $\mathscr{A}$ has the extremal coupled fixed points in $[\alpha, \beta], v_{*}, v^{*}$. Moreover, they satisfy the following characterization:

$$
\left(v_{*}, w^{*}\right)=\min _{\leq}\left\{(v, w):\left(A_{*}(v, w), A^{*}(w, v)\right) \preceq(v, w)\right\},
$$

where

$$
(v, w) \preceq(\bar{v}, \bar{w}) \Longleftrightarrow v \leq \bar{v}, w \geq \bar{w} .
$$

\section{Main Result}

Now, we develop our generalized monotone method applied to problem (1). To do this, throughout this section, we will assume the following.

$\left(H_{1}\right)$ There exists a closed interval $J \subset \mathbb{R}$, such that for a.a. $t \in I$ and all $x \in \mathbb{R}$ the function $f(t, x, \cdot)$ is of bounded variation on $J$. 
Assumption $\left(H_{1}\right)$ implies that there exists a nondecreasing function, $g$, and a nonincreasing one, $h$, such that

$$
f(t, x, \cdot)=g(t, x, \cdot)+h(t, x, \cdot),
$$

for all $(t, x) \in I \times \mathbb{R}$.

Now, we define what we mean by lower and upper solutions for problem (1).

Definition 6. One says that $\alpha, \beta \in \mathscr{C}[a, b+r]$ are, respectively, a lower and upper solutions for problem (1), and if $\alpha_{\mid I}, \beta_{\mid I} \in$ $A C(I)$,

$$
\left[\min _{t \in[a, b+r]} \alpha(t), \max _{t \in[a, b+r]} \beta(t)\right] \subset J,
$$

the compositions

$$
t \longmapsto f(t, \alpha(t), y), \quad t \longmapsto f(t, \beta(t), y)
$$

are measurable for all $y \in J$ and the following inequalities hold:

$$
\begin{gathered}
\alpha^{\prime}(t) \geq g(t, \alpha(t), \beta(\tau(t)))+h(t, \alpha(t), \alpha(\tau(t))), \\
\quad \text { for a.a. } t \in I, \\
\alpha(t) \leq \phi(t), \quad \forall t \in[b, b+r], \\
\beta^{\prime}(t) \leq g(t, \beta(t), \alpha(\tau(t)))+h(t, \beta(t), \beta(\tau(t))), \\
\quad \text { for a.a. } t \in I, \\
\beta(t) \geq \phi(t), \quad \forall t \in[b, b+r] .
\end{gathered}
$$

Remark 7. Notice that, under the previous definition, the lower and the upper solutions appear "coupled." On the other hand, it is assumed that

$$
\min _{t \in[a, b+r]} \alpha(t) \leq \max _{t \in[a, b+r]} \beta(t) .
$$

This is not a strong assumption, taking into account that, as usual, we will ask the lower and the upper solutions to be well ordered in the whole interval $[a, b+r]$.

On the other hand, the fact that $t \mapsto f(t, \alpha(t), y)$ and $t \in$ $I \mapsto f(t, \beta(t), y)$ are being measurable for all $y \in J$ implies that the compositions

$$
\begin{aligned}
& t \in I \longmapsto g(t, \alpha(t), \beta(\tau(t)))+h(t, \alpha(t), \alpha(\tau(t))), \\
& t \in I \longmapsto g(t, \beta(t), \alpha(\tau(t)))+h(t, \beta(t), \beta(\tau(\mathrm{t})))
\end{aligned}
$$

are measurable too, because $g$ and $h$ are being monotone with respect to their last variables.

As we said in the Introduction, an essential tool in our work is the use of coupled quasisolutions. So, we introduce now this concept.

Definition 8. One says that two functions $x_{*}, x^{*} \in$ $\mathscr{C}[a, b+r]$ are coupled quasisolutions of problem (1), and if $x_{* \mid I}, x_{\mid I}^{*} \in A C(I), x_{*}(t)=x^{*}(t)=\phi(t)$ for all $t \in[b, b+r]$ and for a.a. $t \in I$, they satisfy

$$
\begin{aligned}
& x_{*}^{\prime}(t)=g\left(t, x_{*}(t), x^{*}(\tau(t))\right)+h\left(t, x_{*}(t), x_{*}(\tau(t))\right), \\
& x^{* \prime}(t)=g\left(t, x^{*}(t), x_{*}(\tau(t))\right)+h\left(t, x^{*}(t), x^{*}(\tau(t))\right) .
\end{aligned}
$$

We say that these coupled quasisolutions are extremal in a subset $\bar{X} \subset \mathscr{C}[a, b+r]$ if $x_{*}, x^{*} \in \bar{X}$ and $x_{*}(t) \leq x_{1}(t), x_{2}(t) \leq$ $x^{*}(t)$ whenever $x_{1}, x_{2} \in \bar{X}$ is another pair of quasisolutions.

We need the following maximum principle related to problems with advance, as an auxiliar tool, for proving our main result. Compare it with [3, Lemma 3.2], [4, Lemma 1].

Lemma 9. Let $\tau: I \rightarrow[a, b+r]$ be a measurable function such that $\tau(t) \geq t$ for a.a. $t \in I$ and assume that $p \in \mathscr{C}[a, b+r]$ such that $p_{\mid I} \in A C(I)$ and satisfies

$$
\begin{gathered}
p^{\prime}(t) \geq K(t) p(t)-L(t) p(\tau(t)) \quad \text { for a.a. } t \in I, \\
p(t)=0, \quad \forall t \in[b, b+r]
\end{gathered}
$$

where $K, L \in L^{1}(I)$ and $L \geq 0$ a.e.

If

$$
\int_{a}^{b}\left(K_{-}(t)+L(t)\right) d t<1
$$

where $K_{-}=\max \{-K, 0\}$, then $p(t) \leq 0$ for all $t \in[a, b+r]$.

Proof. Let $t_{1} \in[a, b+r]$ such that

$$
p\left(t_{1}\right)=\max _{t \in[a, b+r]} p(t)
$$

and assume by contradiction that $p\left(t_{1}\right)>0$. Then, $t_{1} \in[a, b)$. Now, let $t_{2} \in\left(t_{1}, b\right]$ such that $p\left(t_{2}\right)=0$ and $p(t) \geq 0$ for all $t \in\left[t_{1}, t_{2}\right]$. Now, integrating $t_{1}$ and $t_{2}$, we obtain

$$
\begin{aligned}
p\left(t_{1}\right) & =-\int_{t_{1}}^{t_{2}} p^{\prime}(t) d t \\
& \leq-\int_{t_{1}}^{t_{2}} K(t) p(t) d t+\int_{t_{1}}^{t_{2}} L(t) p(\tau(t)) d t \\
& \leq p\left(t_{1}\right) \int_{t_{1}}^{t_{2}}\left(K_{-}(t)+L(t)\right) d t,
\end{aligned}
$$

and then condition (49) provides the contradiction $p\left(t_{1}\right)<$ $p\left(t_{1}\right)$.

The main result on this paper concerns the existence of extremal quasisolutions and solutions for problem (1). It is as follows.

Theorem 10. Assume $\left(H_{1}\right)$ and that there exist $\alpha, \beta \in \mathscr{C}[a, b+$ $r$ ] which are, respectively, lower and upper solutions for problem (1) such that $\alpha(t) \leq \beta(t)$ for all $t \in[a, b+r]$ and

$$
E=\left[\min _{t \in[a, b+r]} \alpha(t), \max _{t \in[a, b+r]} \beta(t)\right] \subset J .
$$


Assume moreover that the following conditions hold:

$\left(H_{2}\right)$ for each $\gamma_{1}, \gamma_{2} \in[\alpha, \beta]=\{\gamma \in \mathscr{C}[a, b+r]: \alpha(t) \leq$ $\gamma(t) \leq \beta(t)$ for all $t \in[a, b+r]\}$, the final value problem

$$
\left(P_{\gamma_{1}, \gamma_{2}}\right)\left\{\begin{aligned}
x^{\prime}(t)= & F_{\gamma_{2}, \gamma_{1}}(t, x(t)) \\
:= & g\left(t, x(t), \gamma_{2}(\tau(t))\right) \\
& +h\left(t, x(t), \gamma_{1}(\tau(t))\right), \quad \text { for a.a. } t \in I, \\
x(b)= & \phi(b)
\end{aligned}\right.
$$

has the extremal solutions in $[\alpha, \beta]$;

$\left(H_{3}\right)$ there exists $\psi \in L^{1}(I,[0,+\infty))$ such that for a.a. $t \in I$, all $x \in[\alpha(t), \beta(t)]$, and all $y_{1}, y_{2} \in$ $[\alpha(\tau(t)), \beta(\tau(t))]$, one has

$$
\left|g\left(t, x, y_{1}\right)+h\left(t, x, y_{2}\right)\right| \leq \psi(t) ;
$$

$\left(H_{4}\right)$ there exists $K_{1}, K_{2}, L_{1}, L_{2} \in L^{1}(I)$ such that $L_{1}$, $L_{2} \geq 0$ a.e. and

$$
\begin{gathered}
g(t, \bar{x}, y)-g(t, x, \bar{y}) \geq K_{1}(t)(\bar{x}-x)-L_{1}(t)(\bar{y}-y), \\
h(t, \bar{x}, \bar{y})-h(t, x, y) \geq K_{2}(t)(\bar{x}-x)-L_{2}(t)(\bar{y}-y)
\end{gathered}
$$

whenever $\alpha(t) \leq x \leq \bar{x} \leq \beta(t)$ and

$$
\begin{aligned}
& \min _{s \in[b, b+r]} \phi(s)-\int_{t}^{b} \psi(s) d s \\
& \quad \leq y \leq \bar{y} \leq \max _{s \in[b, b+r]} \phi(s)+\int_{t}^{b} \psi(s) d s .
\end{aligned}
$$

Moreover,

$$
\int_{a}^{b}\left(K_{-}(t)+L(t)\right) d t<1
$$

where $K=K_{1}+K_{2}, L=L_{1}+L_{2}$ and $K_{-}(t)=$ $\max \{-K(t), 0\}$.

In these conditions, problem (1) has a unique solution in $[\alpha, \beta]$.

Proof. We consider the space $X=\mathscr{C}[a, b+r]$ endowed with the ordering

$$
\gamma_{1} \leq \gamma_{2} \Longleftrightarrow \gamma_{1}(t) \leq \gamma_{2}(t), \quad \forall t \in[a, b+r],
$$

and we define a multivalued operator

$$
\mathscr{A}:[\alpha, \beta] \times[\alpha, \beta] \subset X \times X \longrightarrow 2^{[\alpha, \beta]} \backslash \emptyset
$$

as follows: for each $\gamma_{1}, \gamma_{2} \in[\alpha, \beta]$, we have $x \in \mathscr{A}\left(\gamma_{1}, \gamma_{2}\right)$ if and only if $x \in[\alpha, \beta], x_{I}$ is a solution of $\left(P_{\gamma_{1}, \gamma_{2}}\right)$ and $x_{\mid[b, b+r]}=\phi$.

Step 1. Operator $\mathscr{A}$ has the extremal coupled fixed points in $[\alpha, \beta]$. By virtue of condition $\left(\mathrm{H}_{2}\right)$, operator $\mathscr{A}$ is well defined and there exist

$$
A_{*}=\min \mathscr{A}\left(\gamma_{1}, \gamma_{2}\right), \quad A^{*}=\max \mathscr{A}\left(\gamma_{1}, \gamma_{2}\right) .
$$

We will show now that $A_{*}, A^{*}$ are mixed monotone and satisfy m.m.c.p. So, let

$$
\gamma_{1}, \bar{\gamma}_{1}, \gamma_{2}, \bar{\gamma}_{2} \in[\alpha, \beta]
$$

such that $\gamma_{1} \leq \bar{\gamma}_{1}, \gamma_{2} \leq \bar{\gamma}_{2}$ and put

$$
x_{1}=A_{*}\left(\gamma_{1}, \gamma_{2}\right), \quad \bar{x}_{1}=A_{*}\left(\bar{\gamma}_{1}, \gamma_{2}\right), \quad \bar{x}_{2}=A_{*}\left(\gamma_{1}, \bar{\gamma}_{2}\right) \text {. }
$$

Then, for all $t \in[b, b+r]$, we have that $x_{1}(t)=\bar{x}_{1}(t)=\bar{x}_{2}(t)$, and for a.a. $t \in I$, we have

$$
\begin{aligned}
\bar{x}_{1}^{\prime}(t) & =g\left(t, \bar{x}_{1}(t), \gamma_{2}(\tau(t))\right)+h\left(t, \bar{x}_{1}, \bar{\gamma}_{1}(\tau(t))\right) \\
& \leq g\left(t, \bar{x}_{1}(t), \gamma_{2}(\tau(t))\right)+h\left(t, \bar{x}_{1}, \gamma_{1}(\tau(t))\right) .
\end{aligned}
$$

And so, $\bar{x}_{1}$ is an upper solution for problem $\left(P_{\gamma_{1}, \gamma_{2}}\right)$. The fact that $x_{1}$ is being the least solution of this problem in $[\alpha, \beta]$ implies that $\bar{x}_{1} \geq x_{1}$ and then $A_{*}\left(\cdot, \gamma_{2}\right)$ is nondecreasing. On the other hand,

$$
\begin{aligned}
x_{1}^{\prime}(t) & =g\left(t, x_{1}(t), \gamma_{2}(\tau(t))\right)+h\left(t, x_{1}(t), \gamma_{1}(\tau(t))\right) \\
& \leq g\left(t, x_{1}(t), \bar{\gamma}_{2}(\tau(t))\right)+h\left(t, x_{1}(t), \gamma_{1}(\tau(t))\right),
\end{aligned}
$$

and therefore $x_{1}$ is an upper solution for problem $\left(P_{\gamma_{1}, \bar{\gamma}_{2}}\right)$. Then, $x_{1} \geq \bar{x}_{2}$, and so the mapping $A_{*}\left(\gamma_{1}, \cdot\right)$ is nonincreasing. In the same way, we show that $A^{*}$ is mixed monotone.

To see that $A_{*}, A^{*}$ satisfy the m.m.c.p., let $\left(v_{j}\right)_{j=1}^{\infty},\left(w_{j}\right)_{j=1}^{\infty}$ be sequences in $[\alpha, \beta]$, one being nondecreasing and the other being nonincreasing. As $A_{*}, A^{*}$ are mixed monotone and bounded, we obtain that the sequences $\left(A_{*}\left(v_{j}, w_{j}\right)\right)_{j=1}^{\infty}$, $\left(A^{*}\left(v_{j}, w_{j}\right)\right)_{j=1}^{\infty}$ have their pointwise limit; say $z_{*}, z^{*}$. As $\left(A_{*}\left(v_{j}, w_{j}\right)\right)_{j=1}^{\infty},\left(A^{*}\left(v_{j}, w_{j}\right)\right)_{j=1}^{\infty}$ are constant in $[b, b+r]$, the convergence is uniform in this interval. On the other hand, for $t, s \in I, s<t$, and $j \in \mathbb{N}$, we have

$$
\begin{aligned}
& \left|z_{j}^{*}(t)-z_{j}^{*}(s)\right| \\
& \leq \int_{s}^{t}\left|g\left(r, z_{j}^{*}(r), w_{j}(\tau(r))\right)+h\left(t, z_{j}^{*}(r), v_{j}(\tau(r))\right)\right| d r \\
& \leq \int_{s}^{t} \psi(r) d r
\end{aligned}
$$

and thus $\left(z_{j}^{*}\right)_{j=1}^{\infty}$ converges to $z^{*}$ uniformly on $I$. The same argument is valid for $z_{*}$.

By application of Theorem 5 , operator $\mathscr{A}$ has the extremal coupled fixed points in $[\alpha, \beta]$; say $x_{*}, x^{*}$.

Step 2. Problem (1) has the extremal quasisolutions in $[\alpha, \beta]$. Indeed, we will show that the extremal coupled fixed points of operators $\mathscr{A}, x_{*}$, and $x^{*}$ correspond with these extremal quasisolutions. First, it is clear that if $x, \bar{x} \in[\alpha, \beta]$ are coupled fixed points of $\mathscr{A}$, then they are coupled quasisolutions of problem (1). On the other hand, if $x, \bar{x}$ are quasisolutions of problem (1), then $A_{*}(x, \bar{x}) \leq x$ and $A^{*}(\bar{x}, x) \geq \bar{x}$, and then 
characterization (9) implies that $x_{*} \leq x$ and $\bar{x} \leq x^{*}$. This shows that $x_{*}, x^{*}$ are the extremal quasisolutions of problem (1) in $[\alpha, \beta]$.

Step 3. Problem (1) has a unique solution in $[\alpha, \beta]$. We will prove this by showing that the extremal quasisolutions $x_{*}, x^{*}$ are, in fact, the same functions, and thus defining a solution of the problem. This solution must be unique in $[\alpha, \beta]$ because if $\bar{x} \in[\alpha, \beta]$ is a solution of (1), then the pair $\bar{x}, \bar{x}$ is also a quasisolution, and then $x_{*} \leq \bar{x} \leq x^{*}$.

To see that $x_{*}=x^{*}$, first notice that as $\left(x_{*}, x^{*}\right)$ is a pair of quasisolutions; then, the reversed pair, $\left(x^{*}, x_{*}\right)$, is quasisolutions too, and then, extremality implies $x_{*} \leq x^{*}$. Moreover, condition $\left(\mathrm{H}_{3}\right)$ implies that for a.a. $t \in I$

$$
x_{*}(t), x^{*}(t) \in\left[\phi(b)-\int_{t}^{b} \psi(s) d s, \phi(b)+\int_{t}^{b} \psi(s) d s\right] \text {. }
$$

Now, define the function $p(t)=x^{*}(t)-x_{*}(t) \geq 0$. On the one hand, $p(t)=0$ for all $t \in[b, b+r]$. On the other hand, condition $\left(H_{4}\right)$ implies for a.a. $t \in I$ that

$$
\begin{aligned}
p^{\prime}(t)= & g\left(t, x^{*}(t), x_{*}(\tau(t))\right)-g\left(t, x_{*}(t), x^{*}(\tau(t))\right) \\
& +h\left(t, x^{*}(t), x^{*}(\tau(t))\right)-h\left(t, x_{*}(t), x_{*}(\tau(t))\right) \\
\geq & K(t)\left(x^{*}(t)-x_{*}(t)\right)-L(t)\left(x^{*}(\tau(t))-x_{*}(\tau(t))\right),
\end{aligned}
$$

and then by virtue of Lemma 9 , we obtain that $p(t) \leq 0$ on $I$. We conclude that $p(t)=0$ for all $t \in[a, b+r]$; that is, $x_{*}=x^{*}$. This ends the proof.

Remark 11. Now, we point out some remarks related to Theorem 10.

(1) Condition $\left(\mathrm{H}_{2}\right)$ could be replaced by any result on the existence of extremal solutions between lower and upper solutions for problem $\left(P_{\gamma_{1}, \gamma_{2}}\right)$. For example, as it is well known, if $F_{\gamma_{1}, \gamma_{2}}$ is a Carathéodory function, then $\left(H_{3}\right)$ implies that $\left(P_{\gamma_{1}, \gamma_{2}}\right)$ has the extremal solutions between $\alpha$ and $\beta$. Moreover, there exists a very extensive literature about the existence of extremal solutions for problem $\left(P_{\gamma_{1}, \gamma_{2}}\right)$ for discontinuous $F_{\gamma_{1}, \gamma_{2}}$. The reader is referred to $[1,8-10]$ and references therein for some results of this type. Notice that although most of these references deal with initial value problems, these results can easily be adapted for final value problems. Finally, notice that $\left(\mathrm{H}_{2}\right)$ implies, in particular, measurability of the composition $t \in$ $I \mapsto F_{\gamma_{1}, \gamma_{2}}(t, x(t))$ for all $x \in[\alpha, \beta]$.

(2) As we said in Section 2, a function of bounded variation has only "jump" discontinuities. Although condition $\left(H_{4}\right)$ implies that for a.a. $t \in I$ the function $f$ is continuous with respect to its third variable in the interval

$$
\begin{gathered}
{\left[\min _{s \in[b, b+r]} \phi(s)-\int_{t}^{b} \psi(s) d s,\right.} \\
\left.\max _{s \in[b, b+r]} \phi(s)+\int_{t}^{b} \psi(s) d s\right],
\end{gathered}
$$

a countable number of discontinuities are allowed to exist outside this interval. Moreover, notice that this interval can be improved if we find another function $\widetilde{\psi}$ satisfying $\left(H_{3}\right)$ and such that $\widetilde{\psi}(t) \leq \psi(t)$ for a.a. $t$.

(3) For almost all $t \in I$ and all $x \in[\alpha(t), \beta(t)]$ the function $f_{t, x}(\cdot)=f(t, x, \cdot)$ is of bounded variation in $[\alpha(\tau(t)), \beta(\tau(t))]$, and thus there exists in this interval a decomposition $f_{t, x}(\cdot)=g_{t, x}(\cdot)+h_{t, x}(\cdot)$, with $g$ nondecreasing and $h$ nonincreasing. Although all conditions in Theorem 10 are stated for an arbitrary Jordan decomposition of this type, all of them can be rewritten with

$$
\begin{gathered}
g_{t, x}(y)=V_{A}^{y}(f), \\
h_{t, x}(y)=f_{t, x}(y)-V_{A}^{y}(f),
\end{gathered}
$$

for any choice of $A \leq \min \{\alpha(t): t \in[a, b+r]\}, A \geq$ $\min J$.

Theorem 10 provides, in particular, a new result on the existence of extremal solutions for problem (1) in the case that function $f$ is nonincreasing with respect to its third variable. In this case, the nondecreasing part of the Jordan decomposition of $f$ does not exist, and therefore the lower and upper solutions introduced in Definition 6 appear uncoupled. Moreover, a pair of quasisolutions in the sense of Definition 8 becomes, in fact, a pair of solutions, and then extremal quasisolutions provided by Theorem 10 reduce to extremal solutions. We specify these ideas in the following corollary.

Corollary 12. Assume that there exist $\alpha, \beta \in \mathscr{C}[a, b+r]$ such that $\alpha_{I}, \beta_{I} \in A C(I), \alpha \leq \beta$ on $[a, b+r]$ and the following inequalities hold:

$$
\begin{array}{r}
\alpha^{\prime}(t) \geq f(t, \alpha(t), \alpha(\tau(t))), \quad \text { for a.a. } t \in I, \alpha(t) \leq \phi(t) \\
\forall t \in[b, b+r], \\
\beta^{\prime}(t) \leq f(t, \beta(t), \beta(\tau(t))), \quad \text { for a.a. } t \in I, \quad \beta(t) \geq \phi(t) \\
t \in[b, b+r] .
\end{array}
$$

Assume moreover that the following conditions hold:

$\left(H_{2}\right)^{\prime}$ for all $\gamma \in[\alpha, \beta]$, the final value problem

$$
\begin{array}{r}
x^{\prime}(t)=f(t, x(t), \gamma), \quad \text { for } a . a . t \in I, \\
x(b)=\phi(b)
\end{array}
$$

has the extremal solutions in $[\alpha, \beta]$; 
$\left(H_{3}\right)^{\prime}$ there exists $\psi \in L^{1}(I,[0,+\infty))$ such that for a.a. $t \in I$, all $x \in[\alpha(t), \beta(t)]$, and all $y \in[\alpha(\tau(t)), \beta(\tau(t))]$, one has

$$
|f(t, x, y)| \leq \psi(t)
$$

$\left(H_{4}\right)^{\prime}$ for a.a. $t \in I$ and all $x \in[\alpha(t), \beta(t)]$, the function $f(t, x, \cdot)$ is nonincreasing.

In these conditions problem (1) has the extremal solutions in $[\alpha, \beta]$.

\section{Delay Problems}

The results obtained in the previous section can be easily reformulated in order to deal with problems with delay. We concrete this idea in the following lines.

Consider the following problem:

$$
\begin{gathered}
x^{\prime}(t)=f(t, x(t), x(\tau(t))), \quad \text { for a.a. } t \in I=[a, b], \\
x(t)=\phi(t), \quad \forall t \in[a-r, a],
\end{gathered}
$$

where $r \geq 0, \tau$ is a measurable function such that $\tau(t) \leq t$ for a.a. $t$; that is, $\tau$ is a delayed argument and $\phi$ is a bounded function which represents the initial state of the solution. Now, by a solution of (43), we mean a function $x \in \mathscr{C}[a-r, b]$ such that $x_{\mid I} \in A C(I)$ and $x$ satisfies both the differential equation (a.e. on $I$ ) and the initial condition.

As we said, we will show now that we can use our technique to obtain a new result on the existence of solutions for problem (43) in the case that function $f(t, x, \cdot)$ is of bounded variation. We begin by reformulating the concept of lower and upper solutions and coupled quasisolutions in order to adapt them to our new problem. As in previous section, we assume $\left(H_{1}\right)$.

Definition 13. One says that $\alpha, \beta \in \mathscr{C}[a-r, b]$ are, respectively, lower and upper solutions for problem (43), and if $\alpha_{\mid I}, \beta_{\mid I} \in$ $A C(I)$,

$$
\left[\min _{t \in[a-r, b]} \alpha(t), \max _{t \in[a-r, b]} \beta(t)\right] \subset J,
$$

the compositions

$$
t \longmapsto f(t, \alpha(t), y), \quad t \longmapsto f(t, \beta(t), y)
$$

are measurable for all $y \in J$ and the following inequalities hold:

$$
\begin{array}{r}
\alpha^{\prime}(t) \leq g(t, \alpha(t), \alpha(\tau(t)))+h(t, \alpha(t), \beta(\tau(t))), \\
\text { for a.a. } t \in I, \\
\alpha(t) \leq \phi(t), \quad \forall t \in[a-r, a], \\
\beta^{\prime}(t) \geq g(t, \beta(t), \beta(\tau(t)))+h(t, \beta(t), \alpha(\tau(t))), \\
\text { for a.a. } t \in I,
\end{array}
$$

Definition 14. One says that two functions $x_{*}, x^{*} \in \mathscr{C}[a-$ $r, b]$ are coupled quasisolutions of problem (43) if $x_{* \mid I}, x_{\mid I}^{*} \in$ $A C(I), x_{*}(t)=x^{*}(t)=\phi(t)$ for all $t \in[a-r, a]$ and for a.a. $t \in I$, they satisfy

$$
\begin{aligned}
& x_{*}^{\prime}(t)=g\left(t, x_{*}(t), x_{*}(\tau(t))\right)+h\left(t, x_{*}(t), x^{*}(\tau(t))\right), \\
& x^{* \prime}(t)=g\left(t, x^{*}(t), x^{*}(\tau(t))\right)+h\left(t, x^{*}(t), x_{*}(\tau(t))\right) .
\end{aligned}
$$

We say that these coupled quasisolutions are extremal in a subset $\bar{X} \subset \mathscr{C}[a-r, b]$; if $x_{*}, x^{*} \in \bar{X}$ and $x_{*}(t) \leq x_{1}(t), x_{2}(t) \leq$ $x^{*}(t)$ whenever $x_{1}, x_{2} \in \bar{X}$ is another pair of quasisolutions.

Before introducing our main result for problem (43), we need a maximum principle for problems with delay, which is as follows. Its proof is analogous to that done in Lemma 9, so we omit it.

Lemma 15. Let $\tau: I \rightarrow[a-r, b]$ be a measurable function such that $\tau(t) \leq t$ for a.a. $t \in I$ and assume that $p \in \mathscr{C}[a-r, b]$ is such that $p_{\mid I} \in A C(I)$ and satisfies

$$
\begin{gathered}
p^{\prime}(t) \leq K(t) p(t)+L(t) p(\tau(t)), \quad \text { for a.a. } t \in I, \\
p(t)=0, \quad \forall t \in[a-r, a],
\end{gathered}
$$

where $K, L \in L^{1}(I)$ and $L \geq 0$ a.e.

If

$$
\int_{a}^{b}\left(K_{+}(t)+L(t)\right) d t<1
$$

where $K_{+}=\max \{K, 0\}$, then $p(t) \leq 0$ for all $t \in[a-r, b]$.

Now, we state our main result in this Section.

Theorem 16. Assume $\left(H_{1}\right)$ and that there exist $\alpha, \beta \in \mathscr{C}[a-$ $r, b]$ which are, respectively, lower and upper solutions for problem (43) such that $\alpha(t) \leq \beta(t)$ for all $t \in[a-r, b]$ and

$$
\widehat{E}=\left[\min _{t \in[a-r, b]} \alpha(t), \max _{t \in[a-r, b]} \beta(t)\right] \subset J .
$$

Assume moreover that the following conditions hold:

$\left(\widehat{H}_{2}\right)$ for each $\gamma_{1}, \gamma_{2} \in[\alpha, \beta]=\{\gamma \in \mathscr{C}[a-r, b]:$ $\alpha(t) \leq \gamma(t) \leq \beta(t)$ for all $t \in[a-r, b]\}$, the initial value problem

$$
\left(\widehat{P}_{\gamma_{1}, \gamma_{2}}\right)\left\{\begin{aligned}
x^{\prime}(t)= & F_{\gamma_{1}, \gamma_{2}}(t, x(t)) \\
:= & g\left(t, x(t), \gamma_{1}(\tau(t))\right) \\
& +h\left(t, x(t), \gamma_{2}(\tau(t))\right), \quad \text { for a.a. } t \in I \\
x(a)= & \phi(a)
\end{aligned}\right.
$$

has the extremal solutions in $[\alpha, \beta]$;

$\left(H_{3}\right)$ there exists $\psi \in L^{1}(I,[0,+\infty))$ such that for a.a. $t \in I$, all $x \in[\alpha(t), \beta(t)]$, and all $y_{1}, y_{2} \in$ $[\alpha(\tau(t)), \beta(\tau(t))]$, one has

$$
\left|g\left(t, x, y_{1}\right)+h\left(t, x, y_{2}\right)\right| \leq \psi(t)
$$


$\left(\widehat{H}_{4}\right)$ there exists $K_{1}, K_{2}, L_{1}, L_{2} \in L^{1}(I)$ such that $L_{1}$, $L_{2} \geq 0$ a.e. and

$$
\begin{aligned}
& g(t, \bar{x}, \bar{y})-g(t, x, y) \\
& \quad \leq K_{1}(t)(\bar{x}-x)+L_{1}(t)(\bar{y}-y), \\
& h(t, \bar{x}, y)-h(t, x, \bar{y}) \\
& \quad \leq K_{2}(t)(\bar{x}-x)+L_{2}(t)(\bar{y}-y)
\end{aligned}
$$

whenever $\alpha(t) \leq x \leq \bar{x} \leq \beta(t)$ and

$$
\begin{aligned}
& \min _{s \in[a-r, a]} \phi(s)-\int_{a}^{t} \psi(s) d s \\
& \quad \leq y \leq \bar{y} \leq \max _{s \in[a-r, a]} \phi(s)+\int_{a}^{t} \psi(s) d s .
\end{aligned}
$$

Moreover,

$$
\int_{a}^{b}\left(K_{+}(t)+L(t)\right) d t<1
$$

where $K=K_{1}+K_{2}, L=L_{1}+L_{2}$ and $K_{+}(t)=$ $\max \{K(t), 0\}$. $[\alpha, \beta]$.

In these conditions, problem (43) has a unique solution in

Proof. The proof is analogous to that done in Theorem 10, but now, redefining operator $\mathscr{A}$ in this way, first, we consider the space $\widehat{X}=\mathscr{C}[a-r, b]$ endowed with the ordering

$$
\gamma_{1} \leq \gamma_{2} \Longleftrightarrow \gamma_{1}(t) \leq \gamma_{2}(t), \quad \forall t \in[a-r, b] .
$$

Then, we consider the operator

$$
\widehat{\mathscr{A}}:[\alpha, \beta] \times[\alpha, \beta] \subset \widehat{X} \times \widehat{X} \longrightarrow 2^{[\alpha, \beta]} \backslash \emptyset
$$

as follows: for each $\gamma_{1}, \gamma_{2} \in[\alpha, \beta]$, we have $x \in \mathscr{A}\left(\gamma_{1}, \gamma_{2}\right)$ if and only if $x \in[\alpha, \beta], x_{I}$ is a solution of $\left(\widehat{P}_{\gamma_{1}, \gamma_{2}}\right)$ and $x_{\mid[a-r, a]}=\phi$.

The rest of the proof is analogous, with obvious changes.

Now, Theorem 16 provides, in particular, a new result on the existence of extremal solutions in the case that function $f$ is nondecreasing with respect to its third variable. For the sake of completeness, we concrete this idea in the following Corollary, which is the analogous to Corollary 12.

Corollary 17. Assume that there exist $\alpha, \beta \in \mathscr{C}[a-r, b]$ such that $\alpha_{I}, \beta_{I} \in A C(I), \alpha \leq \beta$ on $[a-r, b]$ and the following inequalities hold:

$$
\alpha^{\prime}(t) \leq f(t, \alpha(t), \alpha(\tau(t))), \quad \text { for a.a. } t \in I, \alpha(t) \leq \phi(t)
$$

$$
\forall t \in[a-r, a],
$$

$\beta^{\prime}(t) \geq f(t, \beta(t), \beta(\tau(t))), \quad$ for a.a. $t \in I, \beta(t) \geq \phi(t)$

$$
\forall t \in[a-r, a] .
$$

Assume moreover that the following conditions hold:

$\left(\widehat{H}_{2}\right)^{\prime}$ for all $\gamma \in[\alpha, \beta]$, the initial value problem

$$
\begin{array}{r}
x^{\prime}(t)=f(t, x(t), \gamma) \quad \text { for a.a. } t \in I, \\
x(a)=\phi(a)
\end{array}
$$

has the extremal solutions in $[\alpha, \beta]$;

$\left(H_{3}\right)^{\prime}$ there exists $\psi \in L^{1}(I,[0,+\infty))$ such that for a.a. $t \in I$, all $x \in[\alpha(t), \beta(t)]$, and all $y \in[\alpha(\tau(t)), \beta(\tau(t))]$, one has

$$
|f(t, x, y)| \leq \psi(t)
$$

$\left(\widehat{H}_{4}\right)^{\prime}$ for a.a. $t \in I$ and all $x \in[\alpha(t), \beta(t)]$, the function $f(t, x, \cdot)$ is nondecreasing.

In these conditions, problem (43) has the extremal solutions in $[\alpha, \beta]$.

\section{Examples of Application}

We finish this work with two applications of our main results.

Example 1. Consider the following problem with advance:

$$
\begin{gathered}
x^{\prime}(t)=f(x(4 t)), \quad \text { for a.a. } t \in I=\left[0, \frac{\pi}{8}\right], \\
x(t)=\phi(t)=\frac{1}{2}\left(x-\frac{\pi}{2}\right) \sin \left(\frac{1}{x-\pi / 2}\right), \quad \forall t \in\left[\frac{\pi}{8}, \frac{\pi}{2}\right],
\end{gathered}
$$

where $f$ is defined as follows: for each $n \in\{1,2, \ldots\}$, we have

$$
f(y)= \begin{cases}\frac{1}{10} y, & \text { if } y \in(2 n-2,2 n-1], \\ \frac{4 n-1}{10}-\frac{1}{10} y, & \text { if } y \in(2 n-1,2 n],\end{cases}
$$

and for $y \leq 0$, we define $f(y)=-f(-y)$.

Defined that way, $f$ is a function of bounded variation in any bounded interval of $\mathbb{R}$. Moreover, $f$ has a countable number of both downwards and upwards discontinuities. We will construct later a pair $(\alpha, \beta)$ of coupled lower and upper solutions for problem (1) such that for all $t \in[0, \pi / 2]$, we have

$$
-\frac{\pi}{2} \leq \alpha(t) \leq \beta(t) \leq \frac{\pi}{2} .
$$

And then, it suffices to consider a Jordan decomposition of $f$ in the interval $[-2,2]$. So, we put $f=g+h$, with

$$
g(y)=V_{-2}^{y}(f)= \begin{cases}\frac{1}{10} y+\frac{2}{10}, & \text { if } y \in[-2,-1), \\ \frac{1}{10} y+\frac{3}{10}, & \text { if } y \in[-1,1], \\ \frac{1}{10} y+\frac{4}{10}, & \text { if } y \in(1,2],\end{cases}
$$


$h(y)=f(y)-V_{-2}^{y}(f)= \begin{cases}-\frac{2}{10} y-\frac{5}{10}, & \text { if } y \in[-2,-1), \\ -\frac{3}{10}, & \text { if } y \in[-1,1], \\ -\frac{2}{10} y-\frac{1}{10}, & \text { if } y \in(1,2] .\end{cases}$

We will show now that the functions $\alpha(t)=t-\pi / 2=$ $-\beta(t)$ are coupled lower and upper solutions for problem (1). First, we have that $\alpha(t) \leq \phi(t) \leq \beta(t)$ for all $t \in[\pi / 8, \pi / 2]$. On the other hand, for a.a. $t \in I$ we have the following.

(i) If $(\pi / 2)-4 t \in(1,2]$, then $4 t-(\pi / 2) \in[-2,-1)$ and then

$$
\begin{aligned}
& V_{-2}^{\beta(4 t)}(f)+\alpha(4 t)-V_{-2}^{\alpha(4 t)}(f) \\
& \quad=\frac{8}{10}\left(\frac{\pi}{2}-4 t\right)=\frac{-8}{10}\left(\frac{\pi}{2}-4 t\right)+\frac{2}{10} \leq 1=\alpha^{\prime}(t), \\
& V_{-2}^{\alpha(4 t)}(f)+\beta(4 t)-V_{-2}^{\beta(4 t)}(f) \\
& \quad=\frac{8}{10}\left(\frac{\pi}{2}-4 t\right)-\frac{2}{10} \geq-1=\beta^{\prime}(t) .
\end{aligned}
$$

(ii) If $(\pi / 2)-4 t \in[0,1]$, then $4 t-(\pi / 2) \in[-1,0]$ and then

$$
\begin{aligned}
& V_{-2}^{\beta(4 t)}(f)+\alpha(4 t)-V_{-2}^{\alpha(4 t)}(f) \\
& =\frac{8}{10}\left(\frac{\pi}{2}-4 t\right)=\frac{-8}{10}\left(\frac{\pi}{2}-4 t\right) \leq 1=\alpha^{\prime}(t), \\
& V_{-2}^{\alpha(4 t)}(f)+\beta(4 t)-V_{-2}^{\beta(4 t)}(f) \\
& =\frac{8}{10}\left(\frac{\pi}{2}-4 t\right) \geq-1=\beta^{\prime}(t) .
\end{aligned}
$$

Then, $\alpha$ and $\beta$ are coupled lower and upper solutions for problem (61), satisfying $\alpha \leq \beta$ on $[0, \pi / 2]$.

Now, we check condition $\left(\mathrm{H}_{3}\right)$. We have for a.a. $t \in I$, all $x \in[\alpha(t), \beta(t)]$, and all $y_{1}, y_{2} \in[\alpha(4 t), \beta(4 t)]$,

$$
\left|g\left(t, x, y_{1}\right)+h\left(t, x, y_{2}\right)\right| \leq \frac{6}{10}+\frac{5}{10}
$$

and thus condition $\left(H_{2}\right)$ is satisfied with $\psi \equiv 11 / 10$.

Finally, notice that for a.a. $t \in I$, it is

$$
\begin{aligned}
& {\left[\min _{s \in[\pi / 8, \pi / 2]} \phi(s)-\int_{t}^{\pi / 8} \psi(s) d s,\right.} \\
& \left.\max _{s \in[\pi / 8, \pi / 2]} \phi(s)+\int_{t}^{\pi / 8} \psi(s) d s\right] \subset[-1,1],
\end{aligned}
$$

and thus condition $\left(H_{4}\right)$ is satisfied with

$$
L_{1} \equiv \frac{1}{10}, \quad L_{2} \equiv 0
$$

By application of Theorem 10, we conclude that problem (61) has exactly one solution in the functional interval

$$
\left[4 t-\frac{\pi}{2}, \frac{\pi}{2}-4 t\right]
$$

In the following example, we consider a practical application of Corollary 17. It involves a modified logistic-type model with delay.

Example 2. Consider a bacterial culture governed by a logistic-type equation of the form

$$
x^{\prime}(t)=r_{1} x(t)(K-x(t)),
$$

where $x$ represents the population in thousands.

To counteract the effects of saturation term, we introduce an electronic mechanism which acts as follows. It counts the number of individuals and then it provides some food that makes the population grow. The amount of food supplied by the machine is proportional to the number of individuals. Moreover, the machine can distinguish only thousands of individuals and it supplies the food with a delay $\tau$ which also depends on time; as time goes by, this delay increases. Therefore, we can model this process with a differential equation of the form

$$
x^{\prime}(t)=r_{1} x(t)(K-x(t))+r_{2}[x(t-\tau(t))], \quad t \in[0,1],
$$

where $r_{1}, r_{2} \geq 0, K>1$, [.] means integer part and $\tau$ : $t \in[0,1] \rightarrow \tau(t) \in[0, t]$ is measurable. We consider the normalized time interval $[0,1]$ for simplicity.

Finally, we consider an initial population of one thousand individuals. Therefore, we deal with the following initial value problem with delay:

$$
\begin{array}{r}
x^{\prime}(t)=r_{1} x(t)(K-x(t))+r_{2}[x(t-\tau(t))], \\
\text { for a.a. } t \in[0,1], x(0)=1 .
\end{array}
$$

We will show now that problem (73) has extremal solutions between suitable lower and upper solutions.

First, notice that $\alpha \equiv 1$ and $\beta \equiv L$, for large enough $L$, are, respectively, lower and upper solutions for the problem. Indeed, for all $t \in[0,1]$, we have

$$
\begin{gathered}
0=\alpha^{\prime}(t) \leq r_{1}(K-1)+r_{2}, \\
0=\beta^{\prime}(t) \geq r_{1} L(K-L)+r_{2} L, \quad \text { for large enough } L .
\end{gathered}
$$


Then, $\alpha$ and $\beta$ are, respectively, lower and upper solutions for problem (73), which moreover satisfy $\alpha(t) \leq \beta(t)$ for all $t \in[0,1]$.

To check conditions $\left(\widehat{H}_{2}\right)^{\prime},\left(\widehat{H}_{3}\right)^{\prime}$, and $\left(\widehat{H}_{4}\right)^{\prime}$, notice that the differential equation in (73) is defined by the function

$$
f(t, x, y)=r_{1} x(K-x)+r_{2}[y] .
$$

First, for each continuous $\gamma$ such that $1 \leq \gamma(t) \leq L$ for all $t \in[0,1]$, the function

$$
f_{\gamma}(t, x)=f(t, x, \gamma)
$$

is the classical logistic function and then the initial value problem

$$
x^{\prime}(t)=f_{\gamma}(t, x(t)), \quad \text { for a.a. } t \in[0,1], x(0)=1
$$

has extremal solutions (in fact, a unique solution) between $\alpha$ and $\beta$. Therefore, condition $\left(\widehat{H}_{2}\right)^{\prime}$ is satisfied.

Finally, as for $x, y \in[1, L]$, the function $f$ is bounded and, moreover, $f$ is nondecreasing with respect to its third variable; we conclude that $\left(\widehat{H}_{3}\right)^{\prime}$ and $\left(\widehat{H}_{4}\right)^{\prime}$ hold.

Therefore, we can apply Corollary 17 to ensure that problem (73) has the extremal solutions between $\alpha$ and $\beta$.

\section{Acknowledgments}

The author wants to thank the reviewers for their pertinent comments, which contribute to improve the quality of this paper. The paper is partially supported by FEDER and Ministerio de Educación y Ciencia, Spain, Project MTM201015314.

\section{References}

[1] R. Figueroa and R. L. Pouso, "Coupled fixed points of multivalued operators and first-order ODEs with state-dependent deviating arguments," Nonlinear Analysis: Theory, Methods \& Applications, vol. 74, no. 18, pp. 6876-6889, 2011.

[2] A. Dyki and T. Jankowski, "Boundary value problems for ordinary differential equations with deviated arguments," Journal of Optimization Theory and Applications, vol. 135, no. 2, pp. 257269, 2007.

[3] R. Figueroa, "Discontinuous functional differential equations with delayed or advanced arguments," Applied Mathematics and Computation, vol. 218, no. 19, pp. 9882-9889, 2012.

[4] T. Jankowski, "Advanced differential equations with nonlinear boundary conditions," Journal of Mathematical Analysis and Applications, vol. 304, no. 2, pp. 490-503, 2005.

[5] T. M. Apostol, Mathematical Analysis, Addison-Wesley, Reading, Mass, USA, 2nd edition, 1974.

[6] E. Hewitt and K. Stromberg, Real and Abstract Analysis, Springer, New York, NY, USA, 3rd edition, 1975.

[7] S. Heikkilä and V. Lakshmikantham, Monotone Iterative Techniques for Discontinuous Nonlinear Differential Equations, vol. 181 of Monographs and Textbooks in Pure and Applied Mathematics, Marcel Dekker, New York, NY, USA, 1994.

[8] E. R. Hassan and W. Rzymowski, "Extremal solutions of a discontinuous scalar differential equation," Nonlinear Analysis: Theory, Methods \& Applications, vol. 37, no. 8, pp. 997-1017, 1999.
[9] R. L. Pouso, "Nonordered discontinuous upper and lower solutions for first-order ordinary differential equations," Nonlinear Analysis: Theory, Methods \& Applications, vol. 45, no. 4, pp. 391406, 2001.

[10] R. L. Pouso, "Necessary conditions for solving initial value problems with infima of superfunctions," Mathematical Inequalities \& Applications, vol. 8, no. 4, pp. 633-641, 2005. 


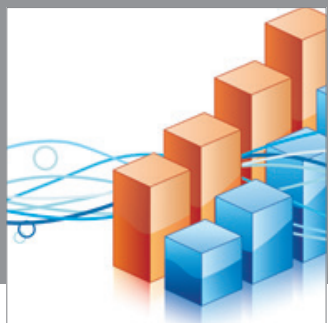

Advances in

Operations Research

mansans

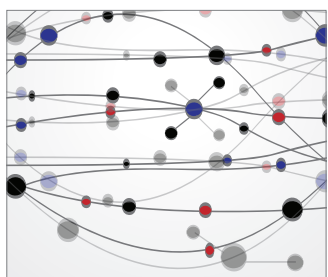

The Scientific World Journal
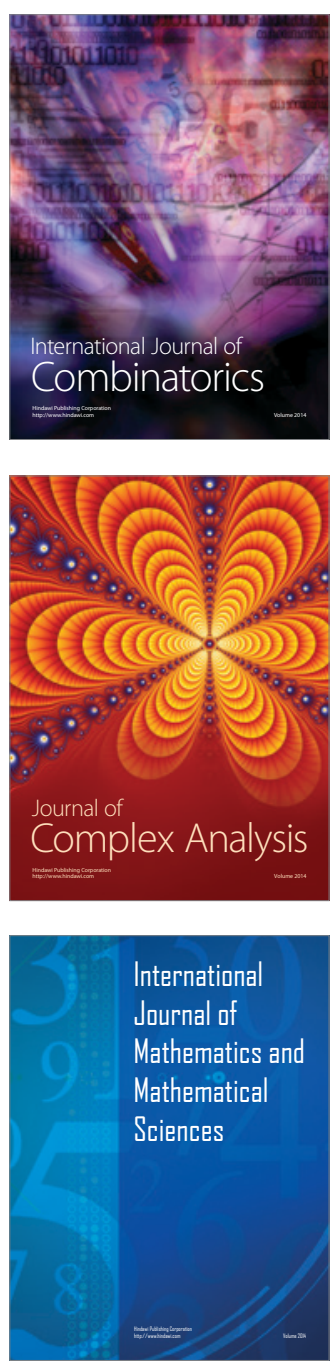
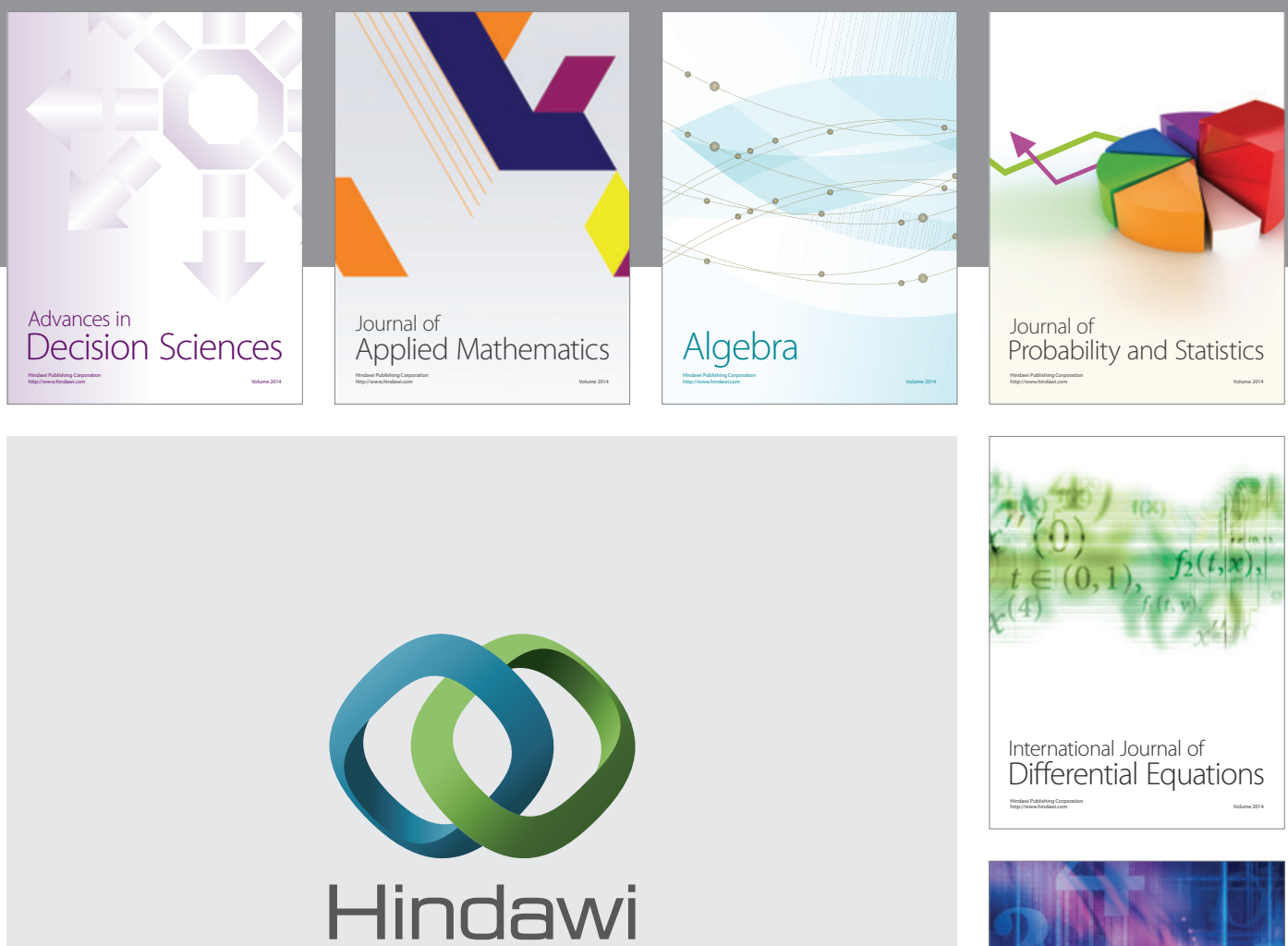

Submit your manuscripts at http://www.hindawi.com
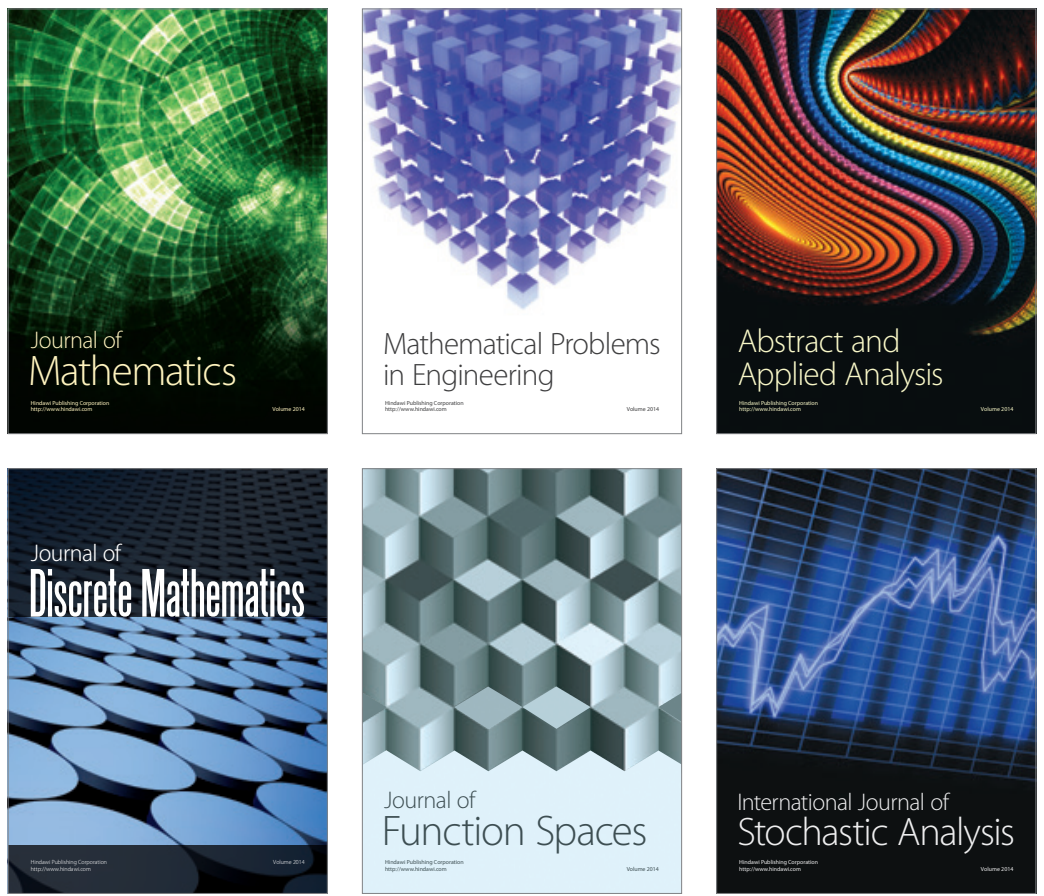

Journal of

Function Spaces

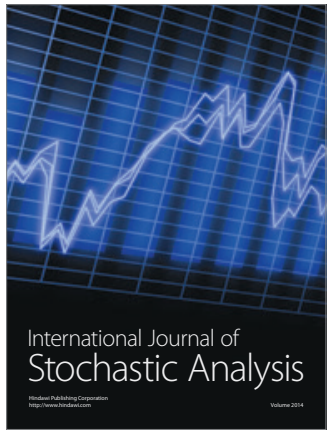

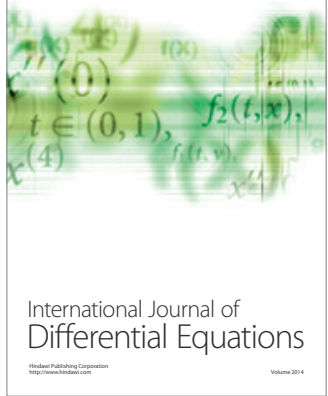
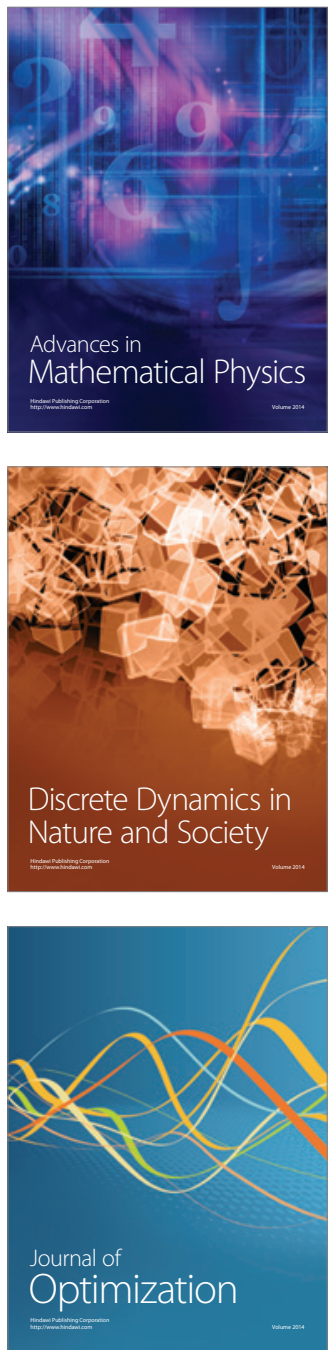